\title{
Derechos de la mujer $y$ derechos universales
}

\section{Women's rights and universal rights}

\author{
VICTORIA CAMPS \\ Universidad Autónoma de Barcelona
}

Recibido: 01/05/00 Aceptado: 28/05/00

\begin{abstract}
RESUMEN
Este trabajo defiende la necesidad, por razones elementales de justicia, de universalizar de manera cabal el ideal de la igualdad de derechos que inspira la concepción de los derechos humanos. Realiza un repaso por varios hitos históricos desde el siglo XIX en una lucha que todavía a finales del siglo siguiente revela que la aspiración a la igualdad de derechos políticos de la mujer sigue siendo un reto pendiente de carácter legal, moral y político.
\end{abstract}

PALABRAS CLAVE

MUJER, DERECHOS HUMANOS, DERECHOS POLÍTICOS, IGUALDAD, FEMI-

NISMO

This work defends the need, for elementary reasons of justice, to fully universalize the ideal of equal rights that inspires the concept of human rights. It reviews several historical milestones since the 19th century in a struggle that still at the end of the 20th century reveals that the aspiration for equal political rights for women continues to be a pending challenge of a legal, moral and political nature.

KEYWORDS

WOMAN, HUMAN RIGHTS, POLITICAL RIGHTS, EQUALITY, FEMINISM

Como es DE SOBRA CONOCIDO, han sido muy pocos los hombres que han apostado decididamente por la igualdad real de los dos sexos. Los textos de filósofos que algo dicen a favor del feminismo son puras excepciones. Por ello no puede

(C) Contrastes. Revista Internacional de Filosofía, vol. XXV-Nº3 (2020), pp. 103-114. ISSN: 1136-4076

Departamento de Filosofía, Universidad de Málaga, Facultad de Filosofía y Letras Campus de Teatinos, E-29071 Málaga (España) 
dejar de sorprender que sea un liberal puro, como lo fue John Stuart Mill, el filósofo que más se haya manifestado a favor de la liberación de la mujer. Es cierto que Mill es un filósofo contradictorio -como todo buen filósofo seguramente. Su utilitarismo no se compadece con la defensa aristocrática y exaltada de la libertad individual, que desarrolla en ese librito magistral e insuperable que es On Liberty. Una doctrina como la utilitarista, según la cual la medida de la justicia es la maximización del bienestar de la mayoría, difícilmente aceptaría poder casi absoluto de las libertades individuales para decidir sobre la propia vida. Hay, en efecto, dos Mill: el utilitarista y el libertario. EI primero es deudor de la influencia de su propio padre y del padre del utilitarismo, Jeremy Bentham. EI segundo es, sin duda, indisociable de la unión con Harriet Taylor. El mismo lo reconoce reiterada, e incluso cansinamente, en su Autobiografía. A los pocos años del primer encuentro con Harriet Taylor, Mill se distancia de los Philosophical Radicals y de las tesis utilitaristas y abraza doctrinas socialistas que ser.in recogidas a su vez por la Fabian Society, germen del laboralismo inglés. El giro hacia el socialismo, ciertamente, se compagina mejor que el utilitarismo con la defensa de las Libertades individuales. Por las mismas fechas, Mill se aleja asimismo de los Philosophic Radicals, de filiación utilitarista y con ambiciones políticas, y se acerca al grupo de los Unitarian Radicals, grupo al que estaba vinculada Harriet Taylor y cuyos intereses eran más sociales y populares y su doctrina más liberal y contraria a las costumbres y moral victorianas.

Es, pues, el Mill radicalmente liberal, pero no liberal a la antigua usanza, el que se pone del lado del reconocimiento de los derechos de la mujer. Lo hace porque no es posible conciliar su concepción individualista de la libertad con una idea que no sea la de la mujer también como individuo libre para decidir sobre su vida. Es en nombre del individualismo que Mill se opone a esa «ley del matrimonio» que da al hombre poderes abusivos sobre la mujer y la mantiene bajo su dominio sin contar para nada con sus deseos ni su voluntad. Es en nombre de la individualidad de cualquier persona -sea hombre o mujer- que argumenta en contra de los prejuicios que están en el fondo de la discriminación de la mujer.

«EI principio que regula las relaciones sociales entre los dos sexos es erróneo en sí mismo, y uno de los mayores obstáculos para el perfeccionamiento humano», tal es la hipótesis y el objetivo de La sumisión de la mujer. EI erróneo principio que ha guiado las relaciones entre hombres y mujeres tiene que ser sustituido por el de la igualdad, que consiste en el no reconocimiento de ningún poder o privilegio a los hombres y de ninguna discapacidad a las mujeres. Todo el texto de Mill da vueltas en torno a esta idea fundamental: ni la supuesta naturaleza ni las costumbres son razón suficiente para que se perpetue la visión de la mujer como el sexo mas debil, indefenso e incapaz. No existe esa «ley 
del más fuerte» que no es sino la coartada para un sinnúrnero de formas de esclavitud y despotismo. El gobierno de los hombres es otra forma de usurpar el poder, auspiciada por una supuesta ley natural y por la generalidad de las costumbres. Una ley más que dudosa y sospechosa, pues, de hecho, hay que decir que nadie está totalmente condicionado por su nacimiento. Esa es, por el contrario, la mayor dificultad que impide el progreso del pensamiento: «la inefable ignorancia y falta de atención de la humanidad a las influencias que forman el carácter humano». La preciosa teoría de Pico della Mirandolla, según la cual el homo ist quamodo omnia está aquí recogida, sin mencionarlo, por el más acérrimo defensor de las libertades del individuo.

Sostiene Mill que la igualdad de derechos haría a las mujeres menos sacrificadas y a los hombres menos egoístas. Es una falacia considerar que en la familia todo es ternura, simpatía y amor: en realidad, la familia es, sobre todo, una escuela de despotismo, donde impera la ley de quien se considera a sí mismo el más fuerte. Mill lo afirma con una sinceridad apabullante: lo único que, en realidad, explica las diferencias entre los dos sexos es que «la generalidad del sexo masculino no puede tolerar la idea de vivir con un igual». Por ello las mujeres necesitan ver reconocido el derecho al sufragio: necesitan protección legal; necesitan, además, leyes no hechas por sus amos, sino por ellas mismas.

Hasta aquí un resumen bastante simple de la defensa de Mill de los derechos de la mujer. De todo ello, lo que ahora me interesa subrayar es lo siguiente: Mill no hubiera llegado nunca a defender tales ideas de no ser por la influencia que tuvo sobre él la tormentosa y, sobre todo, amorosa relación mantenida durante una buena porción vida con Harriet Taylor. Es ella, según la confesión del mismo John Stuart Mill, quien está detrás de la mayoría de sus obras, y no sólo las de carácter feminista. Detrás, no en el sentido en que aún se menciona la absurda obviedad de que «detrás de todo gran hombre... etcétera», sino en el sentido material e intelectual del concepto. Mill no tiene reparo ninguno en admitirlo en su Autobiografía: es Harriet -dice- quien ha inspirado mayormente libros como On Liberty y Political Economy. Ambos son el producto de un joint effort. Dato evidentemente injusto al lado del hecho de que luego es sólo el nombre de Mill el que figura como autor de las obras redactadas y pensadas al unísono. Es la razón y la moral victorianas, que ni el uno ni el otro alcanzan a eludir, lo que les impide tanto hacer público su amor, como firmar las obras que, al parecer, van gestando en común. Por ello no es disparatado pensar que el giro que distancia a Mill de la doctrina utilitarista y lo vuelve más sensible a las cuestiones sociales, así como a la defensa sin paliativos de las libertades del individuo se deben a ideas que Harriet Taylor va metiendo, una a una y repetidamente, en la mente de su amado John Stuart Mill. Es, dicho de otra forma,la innegable concreción fáctica de la esclavitud de la mujer -de la que Harriet es testimonio constante y vivo- lo que le hace 
caer en la cuenta de que el primer e inalienable principio es el de la libertad del individuo, y que ese principio debe incluir explícitamente a la mujer. Una libertad que se contradice con el poder que se han atribuido algunos de decidir sobre la vida de los demás. Contra ese poder se pronuncia Harriet Taylor en Enfranchisement of Women con la claridad y La contundencia de estas Líneas: «Negamos el derecho de una parte de la humanidad a decidir por la otra, el derecho de un individuo a decidir por otro individuo, que es y que no es la 'esfera propia'».

Emilia Pardo Bazán que prologó entusiasmada la traducción al español del texto La sumisión de la mujer, explica cómo, a través de dicho texto, descubrió a John Stuart Mill, filósofo al que tiene por una rara avis en un país como el británico, demasiado pragmático y apegado a la realidad concreta para elevarse desde la facticidad de los hechos a la teoría filosófica. Yo diría que fue precisamente ese pragmatismo de sentido común, la urgencia de rechazar la esclavitud concreta que visiblemente estaba padeciendo la mujer -no la mujer en abstracto, sino su mujer, Harriet Taylor-, lo que verdaderamente condujo a Mill hasta la teoría sobre la libertad. Lo que le llevó a ser un pionero en el reconocimiento de los derechos de la mujer en nombre del individualismo bien entendido.

He traído a colación, y como preámbulo a mi intervención, la situación de John Stuart Mill porque la considero como un ejemplo evidente de que sólo a partir de lo concreto es posible abordar, defender y ensanchar el contenido de los derechos universales. Es conocida la crítica que, desde hace tiempo, viene haciendo la filosofía feminista al liberalismo impulsor de los derechos humanos y, muy en especial, los derechos de la libertad. La misma evolución de los derechos humanos, desde los civiles a los políticos y de estos a los sociales, revela algo innegable: que las libertades individuales son un derecho muy débil y vulnerable cuando faltan la igualdad y la solidaridad. Dicha debilidad ha sido repetidamente criticada por una serie de filósofos, desde Hegel hasta, precisamente, las actuales filósofas feministas. Criticada bajo el reproche de que los derechos a las libertades, que constituyen el núcleo de las primeras declaraciones de derechos, son derechos formales, como lo es el individualismo que les sirve de base. La defensa de las libertades individuales y de unas garantías jurídicas para todos excluye de hecho a aquellos que más reconocimiento necesitan porque no son sujetos políticos con derecho a voto: los no propietarios y las mujeres. La máxima del derecho natural que proclama que «todos los hombres nacen libres e iguales» es empíricamente falsa. No es de extrañar, pues, que las mujeres empiezan su lucha por la libertad reivindicando el derecho al sufragio, el más necesario para ser reconocidas como ciudadanas reales y poder gozar, así, de las libertades que, en teoría, ya eran suyas. No 
es de extrañar tampoco que en esa lucha por el voto, las mujeres se unieran al mismo tiempo a las causas sociales más estridentes, como el abolicionismo o el socialismo utópico. Es la condición de desigualdad radical la que pone de manifiesto la trampa que encierra la proclamación universal del derecho a la libertad.

La Declaración de Séneca Falls, en 1848 -el mismo año en que se publica el Manifiesto Comunista-, marca el comienzo de una serie de reivindicaciones que serán ya imparables. EI mismo precepto lockeano que sirve de base a la Declaración de Independencia de Estados Unidos y que se invoca contra el tráfico, venta y tenencia de esclavos, es reclamado por las mujeres como afirmación de su libertad: «decidimos que todas las leyes que impidan que la mujer ocupe en la sociedad la posición que su conciencia les dicte, o que la sitúan en una posición inferior a la del varón, son contrarias al gran precepto de la naturaleza y, por lo tanto, no tienen fuerza y autoridad».

Europa había visto nacer el liberalismo y la independencia de Estados Unidos se había constituido a la sombra de las doctrinas liberales. Las declaraciones de derechos se habían ido sucediendo unas a otras desde el siglo XVII, ampliándose, de esta manera, el ámbito de las libertades formalmente reconocidas. Pero ahí estaba el problema. EI liberalismo era formal, como lo era el individualismo que lo sustentaba. Universal de iure, pero discriminador e injusto de facto. La mujer era mujer y esposa antes que individuo o ciudadana. Lo peor es que a nadie parece extrañarse el equívoco. Incluso Condorcet, uno de los poetas ilustrados sensibles a la causa femenina, no tuvo reparo ninguno en defender el sufragio censitario. Aceptaba que los propietarios pudieran ser considerados, «sin injusticia», como los únicos ciudadanos del estado.

EI liberalismo, el individualismo y el capitalismo, todo al unísono y en feliz compañía, constituyen los cimientos del sistema patriarcal que se consolida con la modernidad. Un sistema que no acaba de desaparecer a pesar de la resolución de la mujer a lo largo de los dos últimos siglos. Las transformaciones jurídicas para propiciar el acceso de la mujer a la educación, al mercado laboral, a la vida pública, las medidas para independizar a las mujeres de la sumisión conyugal, no han sido suficientes para acabar con la división sexual del trabajo o, como se prefiere calificarla en la filosofía feminista, la división entre la vida privada y la vida pública, una división consagrada por el sistema patriarcal y el liberalismo que lo bendice.

¿Qué tiene de malo tal división? Básicamente dos cosas. En primer término, un reparto del trabajo que mantiene a la mujer sujeta a las llamadas tareas de «cuidado», que son las de la vida doméstica. Tareas o trabajos no reconocidos como tales «trabajos» sencillamente porque no son asalariados. Pero trabajos imprescindibles en una sociedad que le debe atender a los niños y que contempla 
impasible cuanto va envejeciendo su población. Que sean las mujeres quienes deban cargar exclusivamente con esa obligación es, obviamente, injusto.

EI segundo aspecto de la división entre lo privado y lo público que pide a gritos una revisión es la mitificación de la vida privada. La vida privada es el reducto de la libertad y del ocio, el espacio que queda a salvo de interferencias externas, en especial, de la del estado, el ámbito donde el individuo puede sentirse auténticamente libre. Por eso Virginia Woolf pedía «Una habitación propia», como metáfora de la libertad femenina. La realidad, sin embargo, es que la vida privada no significa lo mismo para las mujeres que para los hombres. El lugar de la reproducción biológica, del servicio al otro, de la benevolencia, es el lugar más propio de la mujer «ama de casa», es decir, inactiva, porque la actividad en ella -decía antes- no cuenta como tal actividad. La vida familiar y doméstica no es, para la mujer, el Jugar de expansión de la libertad, sino por el contrario un lugar de privaciones y de dependencia, un obstáculo permanente para la dedicación a otras actividades más rentables. La vida reproductiva no es «productiva».

La constatación de la división sexual del trabajo y de la persistencia de esos dos ámbitos que aún esclavizan a la mujer, ha llevado al feminismo a proponer la necesidad de un cambio de paradigma. Pues no es suficiente reconocer a la mujer como sujeto de los derechos universales. Ese reconocimiento no funciona cuando la situación de la mujer sigue siendo desigual e injusta. No funciona porque sencillamente se deja de ver que las mujeres, en realidad, han sumado unos quehaceres a otros, no han sustituido su vida anterior por otra vida distinta, más parecida a la de los hombres.

Un ejemplo de tal ceguera lo tenemos en uno de los filósofos más sobresalientes de nuestro tiempo, el teórico de la justicia, John Rawls. En el Liberalismo político, Rawls hace una afirmación que ha merecido la repulsa inmediata de las filósofas de la política. «Presumo -afirma Rawls- que la familia es justa». Ya en la Teoría de la justicia, Rawls había cometido el error de pensar en las partes de la posición original como «cabezas de familia». Es más: de la familia dice que es «una asociación pequeña, caracterizada normalmente por una jerarquía definida, en la que cada miembro tiene unos derechos y unos deberes», y añade que la socialización del niño incluye el aprendizaje de los valores del buen esposo, esposa, amigo, ciudadano, etcétera. La familia -la familia realmente existente, hay que suponer- es una escuela de socialización y desarrollo moral. Es decir, pese al trascendentalismo de la posición original que es garantía de imparcialidad, en cuanto Rawls se mete en consideraciones concretas, como la de la familia, pone de manifiesto que su punto de vista no es neutro: es, por el contrario, el de un «él» que quisiera ser neutro pero no puede serlo. Si Rawls hubiera sido una mujer, seguramente los principios de la justicia o los bienes 
básicos deducidos de la supuesta posición original hubieran sido otros (véase el trabajo de Susan M. Okin, «Justice As Fairness - For Whom?», de 1987). Ningún grupo puede hablar en nombre del interés común. Es un error pensar que somos capaces de abstraernos de nuestra condición y colocarnos en el punto de vista moral, que es el de la imparcialidad o el de la racionalidad. Por eso es preciso añadir distintas perspectivas a la perspectiva dominante, para que esta claudique ante otras formas de ver la realidad y enriquezca y amplíe su propia visión. EI solo hecho de que el trabajo ejecutado por mujeres, en la vida privada, haya sido un trabajo socialmente no reconocido es la prueba más evidente de que el hacer de los menos favorecidos solo merece una interpretación: es el hacer del dependiente, del sometido, del esclavo. Un hacer, por consiguiente, que sólo merece desaparecer si de veras queremos liberar al oprimido.

Ante dicha realidad, tenemos dos opciones: 1) eliminar el trabajo que oprime; 2) repartir equitativamente también ese trabajo. La primera opción no sólo es imposible, dudo que realmente la queramos. Por muy bien que se cubran las funciones del «cuidado» convirtiéndolas en servicios públicos o en mercancía privada sometida a la ley de la oferta y la demanda, siempre quedará un cuidado que gratuitamente tendremos que dispensarnos unos a otros. La solución no está en hacer desaparecer cientos de trabajos, sino repartirlos equitativamente. Para conseguirlo, sin embargo, se impone un cambio más radical que el habido hasta ahora, un cambio de paradigma o un nuevo giro copernicano capaz de ver el mundo desde la perspectiva de ambos sexos.

Kant pensó en Copérnico cuando propuso una nueva forma de encarar $\mathrm{La}$ teoría del conocimiento: partamos del hombre y no de las cosas externas a él. El punto de vista desde el que se conoce es fundamental para el conocimiento puesto que de él depende la descripción que hagamos de la realidad. También el feminismo ha querido introducir un nuevo punto de vista, no androcéntrico, que es la perspectiva de «género». Puesto que durante siglos se ha estado hablando del ser humano en general, pero lo han hecho hombres solos, no pudiendo evitar, en consecuencia, su perspectiva androcéntrica, intentemos pensar más específicamente, desde cada uno de los géneros. No ocultemos nuestra realidad ni callemos sobre nosotras mismas. De lo contrario, lo que tengamos que decir se verá rápidamente absorbido por el discurso dominante y ya existente, que no es el nuestro ni el que habla de nuestras necesidades.

¿Cómo incorporar la perspectiva de género? ¿Cómo introducir la perspectiva de la mujer -de lo privado, de hecho- en la vida social, política, incluso la familiar? Hay dos formas de hacerlo. Mejor, una for ma que puede degenerar en otra. Un ejemplo de lo que quiere ser la perspectiva de género son los estudios de la mujer como un aspecto nuevo y distinto de los estudios superiores. Con los estudios de la mujer se trata, precisamente, de corregir las perspectivas 
parciales, sectoriales, androcéntricas, de las ciencias sociales, humanas o incluso las ciencias empíricas. No solo las mujeres que algo han hecho en el mundo de la literatura, del arte, de la filosofía, de la física, están raramente presentes en la historia de todas estas disciplinas -que haya pocos ejemplos de mujeres no es excusa para que, por lo menos, esos ejemplos se subrayen como casos extraordinarios- no sólo, pues, la mujer es ignorada, sino que la forma de narrar, describir, pintar o imaginar la realidad ha tenido que hacerse,sin duda, desde una mirada parcial y prominentemente masculina. Es una información poco discutible: la vida de los hombres y la de las mujeres ha discurrido por territorios y cauces distintos, sus culturas no son las mismas, tampoco sus intereses ni sus necesidades. Esas diferencias marcan inevitablemente la construcción de las realidades de unos 'y otras. Una construcción que, desde hace tiempo, sabemos que no es neutra, ni puro reflejo de algo ya existente. Al conocer, interpretamos $\mathrm{y}$, al interpretar, modificamos lo visto y experimentado.

Con dicho propósito en mente, los estudios de género han querido fijarse, primero, en las mujeres que han tenido un cierto protagonismo en el mundo de los hombres; en segundo lugar, los estudios de la mujer quieren poner de manifiesto la realidad silenciada e ignorada por la visión masculina que llamamos androcéntrica. Cierto que, en el intento, tales estudios corren el peligro de exagerar, pasarse de rosca, rozar el ridículo y no contribuir a presentar una perspectiva diferente, sino sustituir lo conocido por trivialidades. Los estudios de género no siempre han sabido mantener el equilibrio entre el acervo cultural tradicional y dominante y el que apunta y se descubre como algo nuevo y diferente. Han abanderado, en ocasiones, el movimiento de lo políticamente correcto, consistente en ignorar al hombre blanco, heterosexual, occidental, cristiano, para hablar en exclusiva de la mujer negra, homosexual, oriental, etcétera. Esa es la deriva que el cambio de paradigma debería evitar: no se trata de sustituir una perspectiva de género por otra que será la del género contrario, sino integrarlas ambas en una perspectiva no excluyente.

¿Cómo hacerlo? De algún modo, el intento cae en el llamado «feminismo de la diferencia» que, como es sabido, se propone ir más allá de esa igualdad a costa de lo que sea, propugnada por el feminismo más primerizo. Las mujeres, al sentirse ya parte de un mundo que nunca contó con ellas, no piden integrarse sin más al mundo masculino, sino labrarlo de otra forma, preservando y no renunciando a eso que no tenemos más remedio que llamar identidad o cultura femenina. EI actual feminismo de la diferencia no es una visión esencialista: intenta descubrir aquello que nunca ha sido visto porque pertenecía al género no dominante. En la época que celebra la multiculturalidad, la tesis se defiende sola. Si toda visión está culturalmente condicionada, ¿por qué no ha de estarlo la cultura masculina? 
Algunas pensadoras han protagonizado, a mi juicio, con acierto, avances en tal sentido. 'Una de ellas es Carol Gilligan, que lo ha hecho subrayando una categoría nueva e importantísima para la ética: la categoría del cuidado. A nadie se le ocurrió antes colocar el valor del cuidado al lado de otro valor que siempre fue básico: el de la justicia. Sin embargo, ambos son igualmente imprescindibles porque se complementan. EI cuidado está más cerca de la amistad, de la compasión, de la solidaridad, de la caridad. Todos ellos han sido valores éticamente ponderados, aunque ninguno tanto como la justicia, una virtud vinculada a la ley, la cual, como dijo Kant en un texto memorable en los anales de la misoginia, nunca fue ni podía ser cosa de mujeres. No propongo que aceptemos resignadamente el despropósito kantiano y busquemos en nuestro interior qué virtud podemos aportar al mundo, similar a la virtud de la justicia pero más nuestra, más propia de mujeres. Ni la justicia, ni el cuidado son virtudes privativas de uno u otro género. Sólo ocurre que los legisladores han sido mayormente los hombres mientras que las cuidadoras, en cambio, han sido solo mujeres. Si lo que buscamos es mezclar los papeles y las funciones y no vincularlas a ningún género, debemos, en primer lugar, poner de manifiesto las funciones existentes pero ocultarlas y exigir para ellas un reconocimiento similar al de los valores ya establecidos. EI cuidado es un valor tan necesario socialmente como la justicia, no sustituible por la justicia. ¿Por qué ignorarlo? Nancy Fraser, en la línea de Carol Gilligan, propone implantar el modelo de «cuidador universal», que el hombre debería copiar de la mujer. Dice que hay que «inducir a los hombres a asemejarse más a lo que la mayoría de las mujeres son actualmente, es decir, personas que básicamente realizan trabajos de cuidado» (Nancy Fraser, Iustitia interrupta). Pedirle al hombre que copie a la mujer en algo que suele hacer mejor que él porque lo ha hecho más, no es pedir gran cosa cuando las mujeres han asimilado sin titubear lo bueno y lo malo de la cultura construida por los hombres.

Helen Fisher, por su parte, acaba de publicar El primer sexo,donde rebate la tesis de Simone de Beuavoir según la cual «la mujer no nace, sino que se hace». «La mujer nace», para Fisher, con una mente diferente. Nace o, lo que viene a ser lo mismo, es el resultado de una adaptación evolutiva que, por la situación que ha tenido que vivir, es distinta de la del hombre. A causa de sus miles de años de actividad polivalente, las mujeres tienen una mente tipo «red» (web thinking), distinta de La mente masculina, que tiende a fijarse en los objetivos concretos (step thinking), en lugar de visualizar campos más amplios. El fundamento de dicha diferencia, a juicio de Fisher, sólo es en parte biológico: la estructura del cerebro de la mujer es diferente a la del hombre, como son diferentes las hormonas masculinas (testosterona) de las femeninas (estrógenos). Pero las diferencias biológicas nunca son totalmente determinantes: hay 
que añadir las diferencias medioambientales que son asimismo hechos básicos en la formación de actitudes. Fisher, sin embargo, no se limita a constatar esa diversidad. Va más allá y afirma que la especificidad femenina la hace especialmente apta para adaptarse al mundo que viene. La flexibilidad, la ambigüedad, la capacidad de cooperación(y no la necesidad de competir), la perspectiva de futuro son características más propias de la mente femenina que de la mente masculina, características que predisponen mejor a la mujer para un mundo en el que es fundamental el trabajo en equipo, comunicación, los servicios (en especial los sanitarios y educativos). Los hombres seguirán disputando en los trabajos técnicos, los cuales, en principio, se les resisten más a las mujeres.

A mi juicio, Fisher se mueve en un terreno que bordea el peligro al que antes me refería como concomitante a la perspectiva de género. El ingrediente biologista, aunque no sea el único elemento explicativo, da a la teoría un aire esencialista que inevitablemente separa a hombres y mujeres adjudicándoles tareas distintas. Lejos de corregir la división sexual del trabajo, que es de lo que se trata con el cambio de perspectiva, lo que hace Fisher es consolidarla. $\mathrm{Da}$, ciertamente, más relieve a las funciones femeninas, pero las separa y distingue de las masculinas.

Es ese peligro, sin duda, el escollo que hace que algunas feministas se revuelvan contra el feminismo que subraya las diferencias y se propone revalorizar a las mujeres como mujeres. Ahí están, por ejemplo, Celia Amorós y Amelia Valcárcel, resistiéndose a reconocer la bondad del pensamiento de género para la causa femenina. Lo que ambas proponen es ir más allá, trascender el «genérico» y pensar sencillamente desde la perspectiva del sujeto. Hablar de hombres y de mujeres es una abstracción. No hay mujeres en general, sino individuos. Recuperemos, pues, lo mejor del pensamiento liberal y pensemos desde el individuo sin excluir a nadie.

La propuesta de Amorós y de Valcárcel sería aceptable si fuera posible. Es una postura inequívocamente ilustrada, la autocrítica de la Ilustración desde ella misma, desde sus mismos ideales y valores universalizables. No obstante, hace ya tiempo que hemos empezado a verle fisuras a la Ilustración. Nietzsche hizo una genealogía de la moral para poner de manifiesto la parcialidad de los valores supuestamente universales. Wittgenstein desistió de encontrar el verdadero lenguaje para perderse en los distintos juegos lingüísticos, ya que la verdad absoluta no es de nadie. Los posmodernos se cebaron en los equívocos del trascendentalismo, el universalismo y los ideales absolutos, para celebrar el relativismo y la falta de fundamentos. Kant, que es el máximo exponente de la Ilustración y del ideal de universalidad para la ética, es víctima de su yo empírico cuando se propone dar un cierto contenido a las fórmulas vacías del imperativo categórico. Es el ejemplo más claro de que siempre se universaliza 
desde algún lugar concreto, no desde una posición no original -como, a su vez, quiere Rawls-, sino desde una posición ya «situada». Las virtudes y los vicios que se le ocurren a Kant cuando quiere ser concreto son demasiado cristianos para ser universalizables. ¿Cómo elaborar, dadas tales dificultades, ese discurso desde un sujeto trascendental no genérico?

En realidad, creo que la crítica a la perspectiva específicamente femenina esconde un temor no siempre explícito. El temor a que tal punto de vista no consista sino en el intento de hacer de la necesidad virtud puesto que, en realidad, nada bueno ni valioso ni reconocible puede extraerse de unas vidas que solo se han visto marcadas por la dominación y la subordinación al otro. ¿Qué valor podemos otorgar al cuidado, el servicio, el desprendimiento y la dedicación a los demás, que es lo que siempre han hecho, por obligación y no por gusto, las mujeres? ¿No será que estas sienten la culpa de eludir lo que siempre vieron como sus responsabilidades y, en un alarde de abstracción filosófica, convierten esos deberes en deberes universales? Tal vez sea así. Pero aunque lo sea, podemos seguir preguntando: ¿será humana una sociedad sin cuidado, sin individuos desprendidos dispuestos a dedicarse a los otros? La respuesta no me cabe duda que será negativa, viniera de quien viniera: necesitamos, en efecto, esos valores. La mujer no puede ni sabe hacer nada sin recordar al mismo tiempo que tiene unos hijos, una familia, unos padres que exigen atención y cuidado. ¿Por qué han de ser obstáculos solo para ella y no para todos? A toda la sociedad le interesa que ciertos cuidados y servicios no desaparezcan. ¿Por qué considerarlos femeninos y no valores del sujeto humano como tal?

No se trataría, pues, de descubrir lo específicamente femenino, sino aquello que aún no ha sido pensado, descubierto o reconocido como valioso cuando, de hecho, lo es. Se trata de aportar una mayor complejidad a nuestros conceptos, porque la realidad es, efectivamente más compleja. No hubiera habido revolución de la mujer si algunas mujeres no se hubieran levantado a luchar por unos derechos que no tenían. Y si esas mujeres no se hubieran puesto a pensar en las causas de la escisión que ellas mismas sufren en su interior y que sufre también una sociedad que no sabe corregir la división sexual del trabajo. No la sabe corregir porque sabe que necesita el trabajo de las mujeres, pero ya no puede exigírselo sólo a ellas. El cuidado es imprescindible, pero deben asumirlo todos, hombres y mujeres. Por eso es preciso cambiar de paradigma.

La familia limita las posibilidades y capacidades de la mujer, las relaciones laborales son aún discriminatorias para las mujeres, la política aun relega a las mujeres a un espacio que es el de la causa femenina. Es decir, la estructura patriarcal sigue en pie. La mujer ha accedido ciertamente a la vida pública. El Estado de bienestar la ha protegido especialmente. Pero falta algo, la transformación no ha ido a las raíces del problema. Falta definir la política y la ciudadanía 
de otra forma, de una forma que integre lo privado en lo público. «Nunca la política se ha definido como el espacio en el que se fijan metas colectivas que provengan de ambos espacios (el privado y el público)», dice Judith Astellarra en un bello artículo sobre el concepto de ciudadanía. Esa definición coja de la política excluye a la humanidad real, que tiene dos sexos y es mezcla de lo privado y lo público, de razón y sentimiento, de justicia y cuidado. También lo que ocurre en la vida privada es un deber ciudadano, no sólo un deber del esposo o de la esposa, de la madre o del padre. Hay que corregir los sesgos del estado de bienestar para que se respeten los derechos también en el ámbito privado.

Dicho brevemente y volviendo al principio: no se construye filosofía alguna mínimamente creíble desde la limitación que Kant se impuso a si rnismo, citando a Bacon de Verulam: De nobis ipsis silemus. Si callamos sobre nosotras mismas no avanzamos en la corrección del formalismo liberal. Y lo que vale para la mujer, vale para cualquier grupo de desfavorecidos: extranjeros, pobres, homosexuales, enfermos. Aquellos cuya vida y cuya visión del mundo no es la normal, pero que luchan precisamente para que llegue a serlo.

Solo podremos avanzar en el respeto a los derechos fundamentales nombrando a aquellos grupos y sectores que aún escapan a esos derechos. En el siglo XIX las mujeres se constituyeron como grupo que reclamaba sus derechos políticos y su lucha ha tenido resultados no por justos menos asombrosos. Dada la espectacularidad del cambio, hoy ya es impreciso hablar de los derechos de la mujer, en general. Hablamos, mejor, de los derechos de las mujeres de Afganistán, de las mujeres islámicas, de las mujeres maltratadas. No todas están pidiendo lo mismo. Pero todas las discriminaciones -afecten a mujeres o a otros colectivos- tienen como causa última unas relaciones sociales que dejan mucho que desear. Porque no aplican los valores que en teoría reconocen o porque no dan prioridad a lo que debería tenerla. 